\title{
How to Implement an Effective Intervention for Breaks during Working Days-A Field Study
}

\author{
V. Wagner*, E. Pfaffstaller, K. W. Kallus \\ Department of Psychology, University of Graz, Graz, Austria \\ Email: ^verena.wagner@uni-graz.at, evi.pfaffstaller@outlook.com,wolfgang.kallus@uni-graz.at
}

How to cite this paper: Wagner, V., Pfaffstaller, E., \& Kallus, K. W. (2017). How to Implement an Effective Intervention for Breaks during Working Days-A Field Study. Psychology, 8, 728-745. https://doi.org/10.4236/psych.2017.85047

Received: January 19, 2017

Accepted: March 28, 2017

Published: March 31, 2017

Copyright $\odot 2017$ by authors and Scientific Research Publishing Inc. This work is licensed under the Creative Commons Attribution International License (CC BY 4.0).

http://creativecommons.org/licenses/by/4.0/

c) (i) Open Access

\begin{abstract}
Following current prognosis, an aging of the working population is expected. A field study was conducted to investigate interventions to strengthen the ability to work of (older) employees. Interventions should support them to recover from stress to maintain performance and work-related motivation. The current paper addresses this interplay and analyzes the effect of three different interventions (standard intervention; interruption-specific intervention; biological rhythm group). Following research questions should be answered: (1) Can the subjective well-being of employees be improved by specific interventions for breaks? (2) Are there differences between older and younger employees' subjective well-being depending on the contents of break interventions? 34 employees of two Austrian public service organizations participated in the field study. $58.82 \%$ of the participants were female. Following the definition of WHO (1993) whereas "aging workers" are defined as workers which are aged 45 years and older, 21 participants belong to the group "aging workers" resp. "older workers". Overall, the study participants participated in the field study for nine weeks (seven measurement times). With regard to the different intervention groups, the results show that additional intervention contents have a positive effect on the subjective well-being of the employees. Furthermore, older employees benefit more from interventions than younger do. The two main research questions can be answered with the results of the study: (1) Specific interventions for breaks are able to improve the subjective well-being of employees. (2) Differences in the subjective well-being between older and younger employees during the intervention can be made visible.
\end{abstract}

\section{Keywords}

Intervention, Breaks, Employees, Field Study

\section{Introduction}

Following the World Health Organization (WHO, 1993) and current prognosis 
(e.g., Statistik Austria, 2014) an aging of the working population is expected. Therefore, it is becoming increasingly important to keep employees healthy and efficient. In addition, in the light of demographic changes, the working world has to adapt to less available younger professionals. Therefore, elderly skilled employees have to be involved more in the working process (Rothwell, Sterns, Spokus, \& Reaser, 2008; Müller, 2011). Therefore, the attitude towards older employees has to change from negative stereotypes like having problems in learning new content, being inflexible, handicapped, conservative and/or less motivated (Fiore, Borella, \& De Beni, 2012) to positive aspects like being skilled workers with (expert) knowledge and a lot of practical experience. Following Semmer and Richter (2004), older employees are able to compensate performance deficits with the help of expertise and a better comprehension of working processes. Furthermore, they compensate performance deficits by working more precisely (Kallus, Schmitt, \& Benton, 2005) and/or by conducting (additional) training (Wild-Wall, Gajewski, \& Falkenstein, 2009). Ideally, elderly will be able to pass on their knowledge to younger employees. Furthermore, a u-shaped relationship between job satisfaction and age is reported in literature: Job satisfaction of young employees decreases at the age of approx. 30 to 40 years to a minimum, followed by an increase with age (Clark, Oswald, \& Warr, 1996; Zacher, Jimmieson, \& Bordia, 2014). However, job satisfaction changes with time. Whereas, job satisfaction of younger employees seems to be more motivated by extrinsic factors like salary, job satisfaction of older employees correlates stronger with intrinsic factors like reputation or a feeling of "being needed" (Kanfer \& Ackermann, 2004). Furthermore, poor quality of work (effort-reward imbalance and low control) and reduced well-being are associated with the intention to retire early from work (Siegrist, Wahrendorf, von dem Knesebeck, Jürges, \& Börsch-Supan, 2006). Regarding the need of recovery, older employees seem to have a stronger need of recovery than younger employees do (e.g., Jansen, Kant, \& van den Brandt, 2002; Kiss, De Meester, \& Braeckman, 2008).

In this sense, interventions that strengthen the ability to work as well as wellbeing during working days become more and more important. Therefore, interventions should support older employees to recover from stress to maintain performance and work-related motivation. The current paper addresses this interplay and analyzes the effect of three different interventions (standard intervention; interruption-specific intervention; biological rhythm group; see also method section for more details).

\subsection{Recovery}

Recovery is required in order to compensate negative consequences of stress, such as mental fatigue and restore conditions to achieve optimum performance (Allmer, 1996). Kallus and Erdmann (1994) recommended devising recovery as dynamic psychophysical events, which includes both basic biological regulation processes at different physiological levels and mental regulation and control processes, up to complex emotions, cognitions, actions and social interactions. 
Kellmann and Kallus (2000: p. 210) characterize recovery as follows: "recovery is a process in time, is related to the type of and duration of stress, depends on a reduction of, a change of, or a break from stress, is individually specific and depends on individual appraisal, ends when the psychophysical state of restored efficiency and homeostatic balance is reached, includes purposeful action (active recovery), as well as automated psychological and biological processes restoring the initial state (passive recovery) and can be described on various levels (e.g., physiological level, psychological level, social level, socio-cultural level, environmental level). Furthermore, recovery processes can be displayed in various organismic subsystems, various sub-processes of recovery can be dissociated and recovery is closely tied to boundary conditions (e.g., sleep, social contact, etc.)." Following Kallus (2016: p. 42) these characteristics show "that recovery is much more than eliminating fatigue or restarting the system".

Recovery can be influenced actively through individual action. This active control includes the consciously positioning of breaks or active regeneration during a workday (Allmer \& Niehues, 1989). How the optimal distribution of a recovery period between two stress phases might look, describes the so called "deactivation-regeneration-reactivation-model" by Eberspächer, Hermann and Kallus (1993). This model is based on the phase model of recovery from Eberspächer (1988). The model consists of three main phases of the recovery process: distancing phase, regeneration phase and orientation phase. The first phase (a) distancing phase serves to achieve distance from the previous stress phase, thus fulfilling a certain follow-up function. The subsequent (b) regeneration phase fulfills a transition and buffer function between the stress phases and should help to ensure the recovery of optimal performance conditions. During the final (c) orientation phase, a preparation for the following stress phase should take place. The orientation phase should help to reactivate the necessary mental and physical functions according to requirements.

\subsection{Disturbance of Breaks}

According to Article 2 of the "Directive 2003/88/EC of the European Parliament and of the Council of 4 November 2003 concerning certain aspects of the organisation of working time" (European Parliament and Council of the European Union, 2003: p. L299/10), working time is defined as "any period during which the worker is working, at the employer's disposal and carrying out his activity or duties, in accordance with national laws and/or practice". Rest period is defined as "any period which is not working time". Breaks allow employees to recover from work, diminish workload effects and restore exhausted resources (Trougakos \& Hideg, 2009). However, if recovery is prevented or interrupted, it can result in severe health impairments (Allmer, 1996). Kallus and Krauth (1995) distinguish between inadequate and impaired recovery. Inadequate recovery is for example, given when the break is too short and the conditions for optimal recovery are missing. A reduced recovery effect is often referred to an impaired recovery (Kallus \& Erdmann, 1994; Kallus \& Krauth, 1995). This is given when 
optimal conditions for adequate recovery are principally available for the person who wants to recover, but the optimal conditions are disturbed by other circumstances like noise, emotional discussions between family members, etc. (Kallus \& Krauth, 1995). If recovery is disturbed by noise, stress reactions can occur which influence physiological (endocrinological, vegetative) and psychological (like anger, harassment, tension) regulatory mechanisms (cardiovascular, metabolism) (Sust, 1996). Also brooding thoughts, anger, worries and anxieties can interfere recovery (Allmer, 1996). If people fail to distance themselves from work (psychological detachment) stressors remain mentally present. This can lead to an increase in negative emotions like nervousness, anxiety and anger (Watson, 1988). Sonnentag and Bayer (2005) showed that successful distancing from work is associated with positive mood and less fatigue. According to Sonnentag, Binnewies and Mojza (2008) psychological detaching from work during nonworking time protects the sense of well-being of employees and maintains work commitment. Other consequences of recovery deficits are poor sleep, emotional exhaustion, psychosomatic complaints (Sluiter, van der Beek, \& Frings-Dresen, 1999), effects on the subsequent performance (Kallus, Eberspächer, \& Hermann, 1992), more days of illness, chronic fatigue, physical complaints and a greater occurrence of cardiovascular diseases (Blasche, 2008). Therefore, an interruption-specific intervention should enable employees to detach better from work, deal better with disturbances during their breaks and in this way enable them to use their breaks in a better way to improve their well-being and reduce fatigue.

\subsection{Biological Rhythm}

Research of chronobiology already started in the first half of the last century when Kleitman and his colleague tested changes of their endogenous rhythm due to missing information about time in a mammoth-cave (Kleitman, 1963). Based on this, a lot of research followed, like the famous "Andechser bunker experiment", where Aschoff and Wever showed that after an adaption phase, participants followed a clear sleep-wake cycle of about 25 hours. Among other things, they observed that also functions like the body temperature had a circadian rhythm-lowest values in early morning, maximum during the evening (Aschoff, 1965). Biological rhythms may also have an impact on the subjective well-being and the performance of people. After Rothgangel (2010) biological rhythms stabilize the functioning of the organism and help him to regenerate. If biological rhythms are disordered over a long period of time, this may lead to an impairment of the immune defense and lead to psychosomatic complaints.

The cognitive and physical performance varies over the day (Hildebrandt, Moser, \& Lehofer, 1998). For example, according to Zulley and Knab (2003), there is an energy peak between 11 and 12 am and between 3 and 4 o'clock in the afternoon (pm), while the daily low is between 1 and $2 \mathrm{pm}$. Therefore, an intervention with chronobiological aspects resp. a biological rhythm-specific intervention, should make employees sensitive for their biological rhythm and help them to integrate supportive actions in their daily work routine, such as an 
awareness to perform demanding tasks in their own performance highs.

As mentioned before, interventions that strengthen the ability to work as well as well-being during working days become more and more important. The aim of the field study presented in this paper was to survey different interventions to support older employees to recover from stress, to improve subjective well-being and to maintain performance and work-related motivation. Based on the results of former research presented before, three different interventions were used: (a) a standard intervention with content based on the "deactivation-regenerationreactivation-model” (Eberspächer, 1988; Eberspächer, Hermann, \& Kallus, 1993) that includes content of how to structure work in general, how to distance from work before a break and how to orientate on the following working task at the end of a break, (b) an intervention that combines content of the standard intervention and additionally deals with disturbances of breaks and (c) an intervention that combines content of the standard intervention and additionally deals with biological rhythms. The main questions that should be answered with the results of the study were: (1) Can the subjective well-being of employees be improved by specific interventions for breaks? (2) Are there differences between older and younger employees' subjective well-being depending on the contents of break interventions?

\section{Method}

A field study with repeated measures was chosen for the study. The field study was conducted in two Austrian public service organizations. The whole study duration was nine weeks.

\subsection{Sample}

34 employees of two Austrian public service organizations participated in the field study. $58.82 \%$ of the participants were female and $41.18 \%$ were male. Mean age of the participants was 45.03 years $(S D=10.30)$, ranging from 22 to 58 years. Following the definition of WHO (1993) whereas "aging workers" are defined as workers which are aged 45 years and older, 21 participants belong to the group “aging workers" resp. "older workers". All participants worked on a monitorbased workplace. Participants were assigned randomly to the three different intervention groups: (a) a standard intervention with content based on the "deactivation-regeneration-reactivation-model" (Eberspächer, 1988; Eberspächer, Hermann, \& Kallus, 1993; standard group [SG]: $n=9$ ), (b) an intervention that combines content of the standard intervention and additionally deals with disturbances of breaks (interruption-specific intervention [IG]: $n=12$ ) and (c) an intervention that combines content of the standard intervention and additionally deals with biological rhythms (biological rhythm group [BR]: $n=13$ ). The different group sizes results due to the possibilities of the different organizations. At the beginning of the analysis it was checked if the different groups differ significantly from each other at measurement time t0 (baseline measurement). Regarding the study variables, no significant differences at to (baseline) were found 
for the three different intervention groups. Therefore, all effects can be attributed to the interventions.

\subsection{Study Design and Procedure}

To answer the two research questions: (1) Can the subjective well-being of employees be improved by specific interventions for breaks? (2) Are there differences between older and younger employees' subjective well-being depending on the contents of break interventions?, the following independent variables were defined $(2 \times 3 \times 4$ repeated measurement design): Age [younger employees (younger than 45 years old), older employees ( 45 years old and older)] and intervention group [standard intervention group (SG), and two groups with supplementary interventions: interruption-specific intervention (IG), biological rhythm group (BR)]. The measurement repetition factor represents the time of measurement. Overall, the study participants participated in the field study for nine weeks. Within these nine weeks seven different measurement times take place ( $t 0$ - t6; weekly with two resting weeks between $t 4 / t 5$ and $t 5 / t 6$ ). The first time of measurement was a baseline measurement (t0). During $\mathrm{t} 1$ and $\mathrm{t} 2$ all participants received a standard intervention. During the following two measurement times $\mathrm{t} 3$ and $\mathrm{t} 4$ participants of the standard intervention group [SG] deepened the content they have learned before, the two groups with supplementary interventions [IG and BR] learned more about disturbances of breaks (interruption-specific intervention [IG]) or about their biological rhythm (biological rhythm group $[\mathrm{BR}])$. The interventions will be described more detailed at the end of the materials section. After t4 the participants had one resting week without intervention, following by measurement time t5 that gives them the possibility to do a retrospective, think about applicability and use in everyday life of the interventions resp. learned content, repeat content from $\mathrm{t} 1 \mathrm{-t} 4$ if necessary, clarify open points and talk about sustainability of the interventions. Followed by another resting week with no intervention, the field study finished with measurement time t6 (final measurement, evaluation). Within this paper four different measurement times are of interest: baseline measurement [t0], first supplementary intervention [t3], second supplementary intervention [t4], and final measurement [t6]. So, for the following analyses a total of 4 measurement times were considered.

The employees completed daily and weekly questionnaire packages. To answer the research questions five different dependent variables (DV) were conducted: DV1: Recovery-Stress State, DV2: Recovery processes (applicable recreation beneficial processes and impaired recovery during working breaks), DV3: Subjective emotional well-being, DV4: Subjective physical symptoms, DV5: Subjective evaluation of perceived fatigue. The used questionnaires will be described more detailed in the materials section. At the beginning of the field study (measurement time $\mathrm{t} 0$ ), the participants filled out the first questionnaire package including all questionnaires as well as a sociodemographic questionnaire to gather biographical data like age, gender, educational background etc. from the 
participants. This measurement time (t0) was used as a baseline measurement for the following analyses. The participants completed the RESTQ-Work 55/7 and the EPF-AP-44 once a week. All other questionnaires were filled out daily respectively at the end of each working day.

\subsection{Materials}

Recovery-Stress State. For the measurement of the recovery-stress state of employees the German version of the Recovery-Stress Questionnaire for Work 55/7 (RESTQ-Work 55/7, Jiménez \& Kallus, 2016) was used. The RESTQ-Work address different aspects of stress and recovery activities and states in the past seven days/nights. The questionnaire comprises 55 items. The sub-dimensions of the RESTQ-Work 55/7 are: Social-Emotional Stress, Performance(-related) Stress, Overall Recovery, Loss of Meaning/Burnout, Leisure/Breaks, Psychosocial Recovery, and Work-related Recovery. The RESTQ reached good results accompanying interventions and can be seen as good tool for moderators of the intervention process (Wagner, Kallus, Jiménez, \& Kellmann, 2016). Responses are based on a seven-point frequency scale ranging from 0 (never) to 6 (always).

Recovery processes. For the measurement of the recovery processes the German Recovery-Process-Questionnaire-AP-44 (EPF-AP-44) from Eibel and Kallus (2008) was used. It determines applicable recreation beneficial processes and impaired recovery during working breaks. The sub-dimensions of the EPF-AP44 are: preparation phase, dissociation phase, regeneration phase-beneficial to recovery processes, regeneration phase-hindrance to recovery processes, orientation phase, recovery state and absence of recovery effect. Responses are based on a seven-point frequency scale ranging from 0 (never) to 6 (always).

Subjective emotional well-being. For the measurement of the current subjective emotional well-being a category adjective checklist (German version) consisting of 24 items (BSKE-24-ak) from Janke, Debus, Kallus, Hüppe and SchmidtAtzert (1986) was used. It assesses the current emotional state multidimensional. The eight different sub-dimensions of the BSKE are: balance, lifted mood, activation, excitement, irritability, anxiety/sadness, de-activation, and extraversion. Responses are based on a seven-point scale ranging from 0 (not at all) to 6 (most intensive).

Subjective physical symptoms. For the measurement of the current subjective physical symptoms, the 24-item German version of the multidimensional physical symptom list (MKSL-24-ak) from Erdmann and Janke (1994) was used. The seven different sub-dimensions of the MKSL are: pain; nausea, cholinergic symptoms; vegetative symptoms; adrenergic symptoms; general physical relaxation; palpitations; flushing, sensation of heat. Responses are based on a seven-point scale ranging from 0 (not at all) to 6 (most intensive).

Subjective evaluation of perceived fatigue. For the measurement of perceived fatigue the German scale for perceived fatigue (SWM) from Kallus \& Eibel (2008) was used. Responses are based on 7 categories and 51 fine adjustments from 0 (not at all fatigued) to 51 (extremely strong fatigued) following the method of 
the category subdivision approach (Heller, 1985).

Interventions. Within the standard intervention, the participants learned content based on the "deactivation-regeneration-reactivation-model" (Eberspächer, 1988; Eberspächer, Hermann, \& Kallus, 1993) that includes content of how to structure work in general, how to distance from work before a break and how to orientate on the following working task at the end of a break. During the weekly interventions, the participants received information and discussed possibilities to include the learned content in normal working days. For each participant, the weekly intervention lasted for half an hour. The participants should try to practice until the next week resp. next intervention. For the two specific interventions (interruption-specific intervention and biological rhythm intervention), a so-called break-rest protocol was used in addition to the questionnaires. The break-rest protocol allows the participants to survey their activity/activities during working breaks, the frequency and moments of the breaks, sources of disturbance during their breaks and the subjectively assessed restfulness of each break. The results of the break-rest protocols were used to receive more applied information for the interruption-specific intervention [IG] and the biological rhythm intervention [BR]. Subject of the interruption-specific intervention [IG] was to determine sources of disturbance of breaks and to work out strategies to deal better with the existing source of disturbance. In line with the previous described standard intervention, the participants learned about the content during the weekly intervention, discussed possibilities to include it in their normal working life and tried to practice until the next week resp. next intervention. Subject of the biological rhythm intervention were chronobiological aspects. The working day of the participants was adapted to their biological rhythm. For example, the participants learned to perform demanding tasks in their own performance highs. Furthermore, they learned to take breaks following their biological rhythm (e.g., frequency and length of breaks, activating vs. relaxing content, etc.). In addition, to determine the so-called chronotype of the participants, the German version of the Morningness-Eveningness-Questionnaire from Horne and Östberg (D-MEQ) from Griefahn, Künemund and Mehnert (2001) was used. This questionnaire gave information about the wake-up and sleeping habits of the participants. The D-MEQ consists of 19 items. The items have different scale formats. Example item: "How easy do you usually get up in the morning? ... 1 (not easy at all) to 4 (very easy)". It divides the people in extreme morning type, extreme evening type and a neutral type. The individual results of the questionnaire were used as supportive measure for the biological rhythm intervention. In line with the two other previously described interventions, the same procedure was used: the participants learned about the content during the weekly intervention, discussed possibilities to include it in their normal working life and tried to practice until the next week resp. next intervention.

\subsection{Statistical Analyses}

The statistical analyses of the data were conducted using the software SPSS for 
Windows. ANOVA for single and multiple factors with repeated measures were chosen as statistical procedure. The analyses were based on a significance level of 5\%. A descriptive data analysis following Abt (1987) was performed. According to Abt (1987) significances can thus be described by a significance level of $10 \%$, which can be regarded as tendency.

\section{Results}

\subsection{Intervention Group Effects}

The results that should help to answer the first research question (1) Can the subjective well-being of employees be improved by specific interventions for breaks?, are presented below.

The analysis of variance with repeated measures show a significant group effect regarding Performance(-related) Stress, $F(2,20)=4.15, p=.031, \eta_{p}^{2}=.293$. The interruption-specific intervention group [IG] showed significantly less Performance(-related) Stress than the standard group [SG] $(p=.049)$. Also the biological rhythm group [BR] showed tendentially less Performance(-related) Stress than the standard group ( $p=.077)$. Following the mean values (see Figure 1; Table 1) the interruption-specific intervention group shows a constant reduction of Performance(-related) Stress over time. Also, the biological rhythm group shows a constant reduction until $t 4$ but after the break to t6 it increases slightly again.

Regarding Social-Emotional Stress a multivariate analysis of variance shows a significant group effect, $F(2,20)=3.69, p=.043, \eta_{p}^{2}=.270$ : The two groups with the additional content (IG and BR) differ tendentially from the standard group (IG: $p=.069$; BR: $p=.057$ ). Both groups (IG and $\mathrm{BR}$ ) tend to show less Social-Emotional Stress than the standard group (SG; see Table 1).

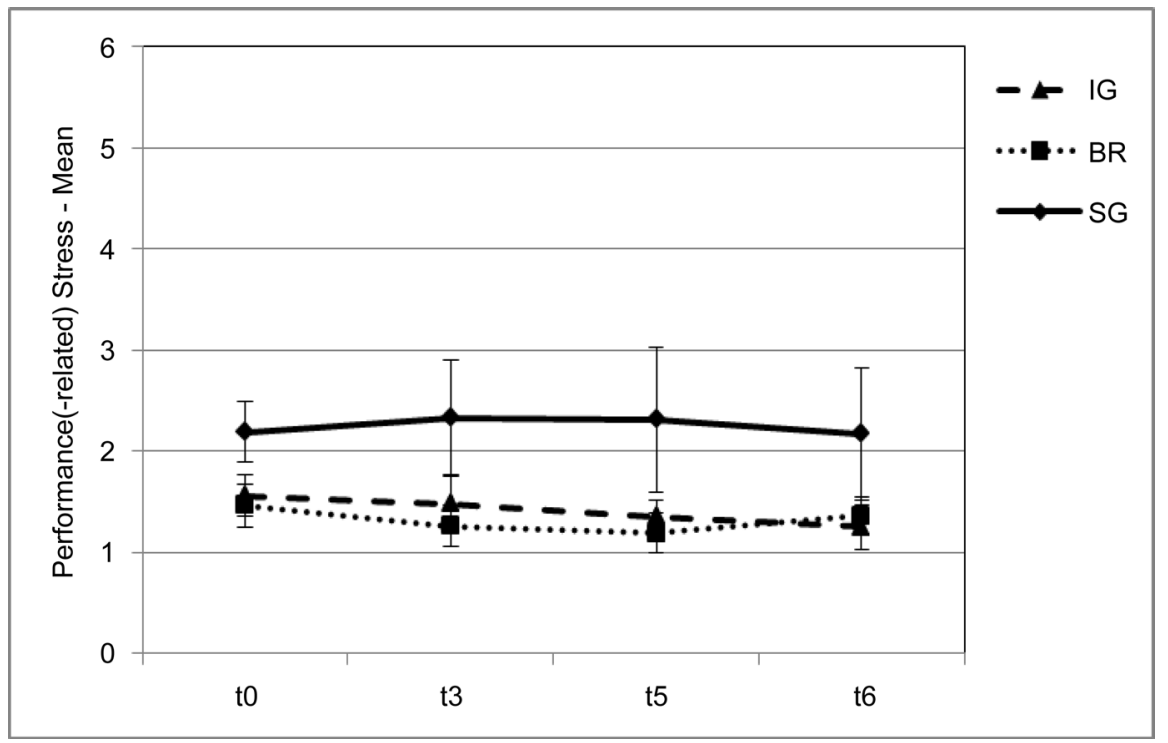

Figure 1. Performance(-related) Stress-Group effect. Note. IG: interruption-specific intervention group; BR: biological rhythm group; SG: standard group; I-I ... Standard error of mean. 
Table 1. Group effects-Mean values and standard deviations.

\begin{tabular}{|c|c|c|c|c|c|c|c|c|c|c|}
\hline \multirow{2}{*}{ Variable } & \multirow{2}{*}{ Sub-Dimension } & \multirow{2}{*}{ Intervention Group } & \multicolumn{2}{|c|}{ t0 } & \multicolumn{2}{|c|}{$\mathrm{t} 3$} & \multicolumn{2}{|c|}{$\mathrm{t} 4$} & \multicolumn{2}{|c|}{ t6 } \\
\hline & & & $M$ & $S D$ & $M$ & $S D$ & $M$ & $S D$ & $M$ & $S D$ \\
\hline \multirow{6}{*}{$\begin{array}{c}\text { Recovery-Stress } \\
\text { State }\end{array}$} & & IG & 1.56 & .69 & 1.48 & .90 & 1.35 & .55 & 1.25 & .72 \\
\hline & Performance (-related) Stress & $\mathrm{BR}$ & 1.46 & .71 & 1.26 & .68 & 1.19 & .63 & 1.36 & .63 \\
\hline & & SG & 2.19 & .49 & 2.33 & 1.18 & 2.31 & 1.38 & 2.17 & 1.29 \\
\hline & & IG & 1.51 & 1.01 & 1.12 & .83 & 1.15 & .81 & 1.14 & 1.00 \\
\hline & Social-Emotional Stress & $\mathrm{BR}$ & 1.50 & .86 & 1.31 & .84 & 1.24 & .85 & 1.04 & .79 \\
\hline & & SG & 2.28 & .74 & 2.28 & 1.07 & 2.31 & 1.68 & 2.17 & 1.26 \\
\hline \multirow{3}{*}{$\begin{array}{l}\text { Recovery } \\
\text { processes }\end{array}$} & & IG & 1.14 & 1.04 & 1.20 & 1.22 & 1.05 & .77 & .93 & .97 \\
\hline & Absence of recovery effect & $\mathrm{BR}$ & .86 & .40 & .94 & .68 & 1.08 & .61 & .86 & .65 \\
\hline & & SG & .90 & .74 & 1.65 & 1.77 & 1.30 & 1.11 & .80 & .76 \\
\hline \multirow{3}{*}{$\begin{array}{l}\text { Subjective } \\
\text { emotional } \\
\text { well-being }\end{array}$} & & IG & 1.87 & 1.01 & 1.54 & 1.14 & 1.49 & .89 & 1.28 & 1.03 \\
\hline & De-activation & $\mathrm{BR}$ & 1.49 & .95 & 1.24 & 1.22 & 1.27 & 1.23 & 1.14 & 1.10 \\
\hline & & SG & 2.00 & .94 & 2.55 & 1.35 & 2.47 & 1.13 & 1.67 & .93 \\
\hline \multirow{6}{*}{$\begin{array}{l}\text { Subjective } \\
\text { physical } \\
\text { symptoms }\end{array}$} & & IG & .26 & .61 & .27 & .64 & .24 & .66 & .36 & .72 \\
\hline & Nausea & $\mathrm{BR}$ & .32 & .80 & .17 & .33 & .17 & .33 & .08 & .21 \\
\hline & & SG & .07 & .15 & .50 & .56 & .58 & .74 & .33 & .53 \\
\hline & & IG & .80 & .54 & .66 & .72 & .56 & .64 & .56 & .68 \\
\hline & Cholinergic symptoms & $\mathrm{BR}$ & .61 & .70 & .40 & .47 & .45 & .39 & .43 & .55 \\
\hline & & SG & .96 & .55 & 1.17 & 1.10 & 1.13 & 1.19 & 1.23 & 1.58 \\
\hline \multirow{3}{*}{\multicolumn{2}{|c|}{ Subjective evaluation of perceived fatigue }} & IG & 18.71 & 9.39 & 20.15 & 12.23 & 17.69 & 8.95 & 11.92 & 8.83 \\
\hline & & $\mathrm{BR}$ & 13.38 & 9.81 & 13.31 & 11.27 & 15.70 & 12.91 & 13.25 & 11.37 \\
\hline & & SG & 23.97 & 1.66 & 26.76 & 11.32 & 26.30 & 9.92 & 22.63 & 8.90 \\
\hline
\end{tabular}

A tendency towards significance can be reported for the effect of the intervention groups for the subjective evaluation of perceived fatigue, $F(2,26)=2.90$, $p=.073, \eta_{p}^{2}=.182$. Considering the effect size the effect is definitely meaningful. Further analysis showed that the biological rhythm group (BR) differs from the standard group (SG; $p=.074$ ). The mean values presented in Table 1 show that the biological rhythm group (BR) tend to experience less fatigue than the standard group (SG).

Regarding Subjective emotional well-being the analysis shows a tendency towards significance for the interaction of time and intervention group in the sub-scale "de-activation", $F(6,78)=1.87, p=.097, \eta_{p}^{2}=.125$. Here again, considering the effect size the effect is quite meaningful. As shown in Table 1 de-activation increases first in the standard group (see $\mathrm{t} 3$ and $\mathrm{t} 4$ by comparison t0), whereas de-activation decreases steadily in the groups with additional intervention (IG and BR).

Regarding Subjective physical symptoms, the analyses showed a significant interaction between time of measurement and group, $F_{H F}(4.18,54.38)=2.57$, 
$p=.025, \eta_{p}^{2}=.17$, for the sub-scale "nausea". The participants of the biological rhythm group reported constantly less nausea, whereas the standard group showed an increase of nausea until t4. The manifestation decreases until t6, but remains, however, over the initial state (see Table 1).

Furthermore, a significant interaction between time of measurement and group regarding the sub-scale "cholinergic symptoms" can be shown, $F_{H F}(5.08$, $66.04)=2.47, p=.040, \eta_{p}^{2}=.160$. The biological rhythm group (BR) reported significantly less physical arousal than the standard group $(p=.007)$. This means that they reported for example less physical weakness or strange feeling in the stomach. Also, the group with the interruption-specific intervention (IG) shows a constant decrease of "cholinergic symptoms" (see Table 1).

The results of the analysis of recovery processes showed that the interaction of time and group, $F_{H F}(4.90,46.57)=1.97, p=.103, \eta_{p}^{2}=.171$ for the sub-dimension "absence of recovery effect" just failed the level of significance. Again, the effect size shows that the effect is definitely meaningful (mean values see Table 1).

All other effects do not reach the level of significance.

\subsection{Age Effects}

To answer the second research question (2) Are there differences between older and younger employees' subjective well-being depending on the contents of break interventions?, age effects were analyzed. The results are as follows.

Regarding Performance(-related) Stress the results show a tendency towards significance for the difference between younger and older employees/participants, $F(3,60)=2.23, p=.094, \eta_{p}^{2}=.100$. During the field study Performance (related) Stress of older employees decreases over time, while the values of the younger participants rise slightly (see Table 2).

Table 2. Age effects-Mean values and standard deviations.

\begin{tabular}{|c|c|c|c|c|c|c|c|c|c|c|}
\hline \multirow{2}{*}{ Variable } & \multirow{2}{*}{ Sub-Dimension } & \multirow{2}{*}{ Age } & \multicolumn{2}{|c|}{ to } & \multicolumn{2}{|c|}{$\mathrm{t} 3$} & \multicolumn{2}{|c|}{$\mathrm{t} 4$} & \multicolumn{2}{|c|}{ t6 } \\
\hline & & & $M$ & $S D$ & $M$ & $S D$ & $M$ & $S D$ & $M$ & $S D$ \\
\hline \multirow{4}{*}{ Recovery-Stress State } & \multirow{2}{*}{ Performance(-related) Stress } & $<45$ years & 1.43 & .75 & 1.56 & 1.11 & 1.53 & 1.06 & 1.56 & 1.03 \\
\hline & & $\geq 45$ years & 1.84 & .63 & 1.63 & .88 & 1.51 & .83 & 1.46 & .83 \\
\hline & \multirow[b]{2}{*}{ Work-related Recovery } & $<45$ years & 3.42 & .93 & 2.90 & .72 & 3.10 & .81 & 2.99 & .98 \\
\hline & & $\geq 45$ years & 3.52 & .74 & 3.68 & 1.06 & 3.63 & 1.16 & 3.74 & 1.05 \\
\hline \multirow{5}{*}{ Recovery processes } & \multirow{3}{*}{ Absence of recovery effect } & $<45$ years & 1.05 & .74 & 1.66 & 1.24 & 1.36 & .83 & 1.05 & .79 \\
\hline & & & & & & & & & & \\
\hline & & $\geq 45$ years & .95 & .84 & .84 & 1.02 & .91 & .68 & .75 & .80 \\
\hline & \multirow{2}{*}{$\begin{array}{l}\text { Regeneration phase-hindrance } \\
\text { to recovery processes }\end{array}$} & $<45$ years & 1.20 & 1.13 & 1.39 & 1.14 & 1.14 & 1.19 & 1.18 & 1.12 \\
\hline & & $\geq 45$ years & .80 & .73 & .80 & 1.08 & .84 & .94 & .63 & .79 \\
\hline \multirow{2}{*}{$\begin{array}{l}\text { Subjective emotional } \\
\text { well-being }\end{array}$} & \multirow{2}{*}{ Cholinergic symptoms } & $<45$ years & .81 & .69 & .82 & 1.02 & .75 & 1.03 & .92 & 1.40 \\
\hline & & $\geq 45$ years & .74 & .56 & .61 & .64 & .61 & .58 & .53 & .59 \\
\hline
\end{tabular}


The analysis show that older employees reported tendentially more Workrelated Recovery than younger participants, $F(1,20)=3.60, p=.072, \eta_{p}^{2}$ $=.153$. Considering the effect size, the effect is definitely meaningful. Younger participants reported less Work-related Recovery (e.g., action latitude, participation and personal growth factors). As shown in Table 2, the initial Work-related Recovery of younger and older participants was quite similar. Following the mean values older employees show an increase of the Work-related Recovery over time, whereas Work-related Recovery of younger employees decreases. The largest enhancement was shown between $\mathrm{t} 0$ and $\mathrm{t} 3$.

A significant interaction between time and age was shown in the sub-dimension "absence of recovery effect" of the Recovery processes, $F_{H F}(2.45,46.57)=4.43$, $p=.012, \eta_{p}^{2}=.189$. Following the mean values (Table 2), younger workers show from $\mathrm{t} 3$ on a stronger "absence of recovery effect" than older employees do. Whereas older worker remain more or less constant over time, younger employees show an increase between $\mathrm{t} 0$ and $\mathrm{t} 3$. After $\mathrm{t} 3$ they register a constantly decrease of the "absence of recovery effect". An additional computed univariate analysis of variance showed a tendency towards significance for the difference between older and younger employees at $\mathrm{t} 3, F(1,23)=3.31, p=.082$, $\eta_{p}^{2}=.126$. Here again, considering the effect size, the effect is quite meaningful. No significant differences could be shown for the two other measurement times $\mathrm{t} 4$ and t6 $\left(\mathrm{t} 4: F(1,23)=2.26, p=.147, \eta_{p}^{2}=.089 ; \mathrm{t} 6: F(1,23)=.85, p=.376\right.$, $\left.\eta_{p}^{2}=.036\right)$.

Regarding the dimension "regeneration phase-hindrance to recovery processes" (impaired relaxation processes and exhaustion) younger participants reported more fatigue and exhaustion than the older participants, $F(1,19)=$ $4.16, p=.056, \eta_{p}^{2}=.180$. Interestingly, fatigue and exhaustion of older employees decreases from $t 4$ to t6 while younger employees experienced a slight increase during the same time (see also Table 2).

Furthermore, a tendency towards significance can be shown for the interaction of time and age regarding the sub-scale "cholinergic symptoms" of Subjective physical symptoms, $F_{H F}(2.54,66.04)=2.57, p=.071, \eta_{p}^{2}=.090$. Whereas older employees showed steadily less physical arousal over time, younger employees remain more or less constant until $t 4$, followed by an increase of their "cholinergic symptoms" at t6 (see also Table 2).

All other effects do not reach the level of significance.

\section{Discussion}

Regarding the expected aging of the working population in the next years (WHO, 1993; Statistik Austria, 2014) and in the light of demographic changes, at the same time less available younger professionals (Müller, 2011) a field study was conducted to investigate interventions to strengthen the ability to work of (older) employees. The field study should help to answer the two research questions: (1) Can the subjective well-being of employees be improved by specific interventions for breaks? (2) Are there differences between older and younger 
employees' subjective well-being depending on the contents of break interventions? The aim was to find out the impact of different contents of interventions on the subjective well-being of older and younger employees.

With regard to the different intervention groups, the results of the field study showed in general that additional contents (interruption-specific intervention [IG], biological rhythm intervention [BR]) compared to the "pure" break intervention (standard group [SG]) have a positive effect on the subjective well-being of the employees. The groups with interventions with additional content (IG, BR) have better values than the standard group. With regard to the recovery-stress state, it could be shown that participants who got interventions with additional content, report less Performance(-related) Stress (e.g., unresolved conflicts, fatigue and lack of energy) as the standard intervention group (IG). Furthermore, it was shown that the groups with additional intervention content report less social-emotional stress, less general and emotional stress, and less social tension than the standard group. These results could be due to the fact that the intervention group "Biological Rhythms" (BR) additionally learned more about daytime performance fluctuations and the fact that the participants of this intervention group tried to adjust their breaks to this. This could have led to an optimization of working time and break design and have thus caused a reduction in the stress faced. The second intervention group (IG) learned more about how to deal with disturbances and interruptions of breaks. Therefore, they should be able to cope better with possible sources of interference in breaks, and thereby make better use of breaks and so reduce stress effects stronger. Regarding recovery, the groups do not differ significantly.

Taking the physical and mental well-being into account, it could be shown again that the two groups with interventions with additional contents (IG, BR) received better results than the standard intervention group (SG). For perceived fatigue the descriptive description of the curves shows that employees with interventions with additional content could benefit more from the intervention than those of the standard group. In particular, comparing the group "dealing with interruptions of breaks" (IG) with other groups, the IG had the strongest reduction of fatigue starting from the measurement time when they received the first additional intervention. Another explanation of these effects may be that the content of the pure break specific intervention (standard intervention group, SG) did not include any new information for the participants, as these interventions greatly covered issues of working time structuring or the distancing and orientation phase in recovery processes. Bamberg, Ducki and Metz (1998) pointed out that employees are the true experts in terms of their working conditions. Perhaps, the pure break specific interventions were rather reminder or refresher as they have novelties character for the study participants, while the additional interventions included new topics that animated them to try out new activities and strategies and integrate them into their everyday lives.

There were also age-specific effects: The subjective physical symptoms showed results with a tendency towards significance for the subtest "cholinergic symp- 
toms". Older study participants reported less physical weakness or a strange feeling in the stomach than younger participants. With regard to the recovery-stress state it was shown that older study participants reported tendentially more Work-related Recovery which means that they tended more to "activitybased resources", as for example, action latitude (in terms of completing tasks), participation (in terms of involvement in decisions) and personal growth factors (in terms of promotion and insertion of own competencies) than younger participants. In addition, older participants appeared to be less tired by the intervention and reported about having more energy and less physical discomfort over time compared to younger participants. Furthermore, for younger participants the stress they faced seems likely to rise over time. At the same time Performance(-related) Stress of older employees decreases over time. Interestingly, younger and older participants described themselves, however, at the end of the field study (measurement time t6) as nearly similar stressed.

Concerning recovery processes, a significant result in dimensional "absence of recovery effect' between measurement time and age can be shown. Compared to the baseline measurement (t0) older employees showed a slight improvement in this dimension to final measurement ( $\mathrm{t} 6$ ). Younger employees, however, felt in comparison to the baseline measurement still exhausted resp. disturbed in their workflow, due to breaks at measurement time t3. Beginning at measurement time $\mathrm{t} 3$, these values improved constantly and reached back up to the starting level until the final measurement $t 6$. Another significant effect of age can be seen in the dimension "regeneration phase-hindrance to recovery processes" (impaired relaxation processes and exhaustion). Younger employees reported from beginning to the end of the field study more recreation obstructive processes than older employees. This means that they could not adequately recover during their breaks due to other circumstances such as fatigue, stress, anger or lack of opportunities to take breaks. For older employees an improvement can be shown between measurement time $t 4$ and $t 6$, while the younger ones reported more fatigue and impaired recovery processes. One explanation would be that the majority of the younger study participants had a job description in which they once or several times a week have to work outside the company to for example visit customers etc. The participants reported that they experienced a lot of time pressure during these times (e.g., appointments, drives from one place to another etc.). They mentioned that the time pressure can be seen as a reason why they experienced marginal possibilities to set breaks during these times. Unfortunately, a comparison group with older employees with similar job description was not available in the field study, but would be of interest for subsequent studies comparing age-specific effects.

In summary following the results, older employees benefit more from interventions than younger do. Following Semmer and Richter (2004) older employees are able to compensate performance deficits by a better comprehension of working processes. The interventions investigated in the field study can support them too. The two main research questions can be answered with the re- 
sults of the study: (1) Specific interventions for breaks are able to improve the subjective well-being of employees. (2) Differences in the subjective well-being between older and younger employees during the intervention can be made visible. However, results of the study do not indicate an interaction between age and contents of break interventions. Obviously, breaks in the working environment cannot only be seen from a behavioral point of view. Rather, companies must contribute to the "break culture" (circumstances-oriented point of view).

\section{Study Limitations and Directions for Future Research}

This study has some limitations. First, the study participants participated in the field study for nine weeks. To measure long-term effects of the interventions, we would suggest including follow-up measurements after for example six month and/or one year in future studies.

Second, it might be interesting if a process with short intervention sessions to refresh the learned content (e.g. every six month, every year etc.) could improve the long-term effects. Further studies should be carried out to investigate this concept.

\section{Acknowledgements}

This work was supported by the Oesterreichische Nationalbank (OeNB) Anniversary Fund (grant number 15025). The authors would like to acknowledge Kerstin Eibel, Vesna Pavlovski and Zsófia Berkes for their collaboration during the research project, and all participants who participated. The authors acknowledge the financial support by the University of Graz.

\section{References}

Allmer, H. (1996). Erholung und Gesundheit: Grundlagen, Ergebnisse und Maßnahmen [Recreation and Health: Basics, Results and Measures]. Göttingen: Hogrefe.

Allmer, H., \& Niehues, C. (1989). Individuelle Erholungsmaßnahmen nach mentalen Arbeitsanforderungen unter Berücksichtigung der sportlichen Aktivität [Individual Recreational Activities According to Mental Work Requirements, Taking into Account the Sporting Activity]. Brennpunkt der Sportwissenschaften, 3, 164-183.

Aschoff, J. (1965). Circadian Rhythms in Man. Science, 148, 1427-1432. https://doi.org/10.1126/science.148.3676.1427

Bamberg, E., Ducki, A., \& Metz, A.-M. (1998). Handbuch Betriebliche Gesundheitsförderung [Handbook of Occupational Health Promotion]. Göttingen: Verlag für Angewandte Psychologie.

Blasche, G. (2008). War Ihr Urlaub erholsam? Ergebnisse und Anwendungen der Erholungsforschung [Was Your Vacation Restful? Results and Applications of RecoveryResearch]. Psychologie in Österreich, 3, 306-314.

Clark, A., Oswald, A., \& Warr, P. (1996). Is Job Satisfaction U-Shaped in Age? Journal of Occupational and Organizational Psychology, 69, 57-81. https://doi.org/10.1111/j.2044-8325.1996.tb00600.x

Eberspächer, H. (1988). Individuelle Handlungsregulation [Individual Action Regulation]. Schorndorf: Hofmann.

Eberspächer, H., Hermann, H.-D., \& Kallus, K. (1993). Psychische Erholung und Re- 
generation zwischen Beanspruchungen. In J. R. Nitsch, \& R. Seiler (Eds.), Bewegung und Sport: Psychologische Grundlagen und Wirkungen 1 (pp. 237-241). Sankt Augustin: Academia.

Eibel, K., \& Kallus, K. W. (2008). Der Erholungs-Prozess-Fragebogen-AP-44 (EPF-AP44) [German Version of the Recovery-Process-Questionnaire-AP-44]. Unpublished Questionnaire, Graz: University of Graz, Department of Psychology.

Erdmann, G., \& Janke, W. (1994). Die mehrdimensionale körperliche Symptomliste (MKSL) [German Version of the Multidimensional Physical Symptom List]. Berlin: TU Berlin.

European Parliament and Council of the European Union (2003). Directive 2003/88/EC of the European Parliament and of the Council of 4 November 2003 Concerning Certain Aspects of the Organisation of Working Time. Official Journal of the European Union, L299/9-L299/19.

Fiore, F., Borella, E., \& De Beni, R. (2012). How Old Are Older Workers? From Age Stereotypes to Successful Intergenerational Relationships. Life Span and Disability, 15, 35-53.

Griefahn, B., Künemund, C., \& Mehnert, P. (2001). Zur Validität der deutschen Übersetzung des Morningness-Eveningness-Questionnaires von Horne und Östberg [Validity of the German Translation of the Morningness-Eveningness-Questionnaires by Horne und Östberg]. Somnologie-Schlafforschung und Schlafmedizin, 5, 71-80. https://doi.org/10.1046/j.1439-054X.2001.01149.x

Heller, O. (1985). Hörfeldaudiometrie mit dem Verfahren der Kategorienunter-teilung (KU) [Listening Field Audiometry by the Process of Categorical Subdivision (KU)]. Psychologische Beiträge, 27, 478-493.

Hildebrandt, G., Moser, M., \& Lehofer, M. (1998). Chronobiologie und Chronomedizin [Chronobiology and Chronomedicine]. Stuttgart: Hippokrates.

Janke, W., Debus, G., Kallus, K. W., Hüppe, M., \& Schmidt-Atzert, L. (1986). BSKE (EWL)-ak [German Version of the Adjective Checklist]. Unpublished Questionnaire, Würzburg: Julius-Maximilians-University Würzburg.

Jansen, N. W. H., Kant, I. J., \& van den Brandt, P. (2002). Need for Recovery in the Working Population: Description and Associations with Fatigue and Psychological Distress. International Journal of Behavioral Medicine, 9, 322-340. https://doi.org/10.1207/S15327558IJBM0904_03

Jiménez, P., \& Kallus, K. W. (2016). Recovery-Stress Questionnaire for Work (RESTQWork). Frankfurt am Main: Pearson Assessment \& Information GmbH.

Kallus, K. W. (2016). RESTQ-Basic: The General Version of the RESTQ. In K. W. Kallus, \& M. Kellmann (Eds.), The Recovery-Stress Questionnaires: User Manual (pp. 49-85). Frankfurt am Main: Pearson Assessment \& Information GmbH.

Kallus, K. W., \& Eibel, K. (2008). Die Skala zur wahrgenommenen Ermüdung [German Version of the Scale for Perceived Fatigue]. Unpublished Questionnaire, Graz: University of Graz, Department of Psychology.

Kallus, K. W., \& Erdmann, G. (1994). Zur Wechselbeziehung zwischen Ausgangszustand, Belastung und Erholung [The Interrelations of Initial Sate, Stress and Recovery]. In R. Wieland-Eckelmann, H. Allmer, J. Otto, \& K. W. Kallus (Eds.), Arbeit und Erholung. Studien zur Aktivierung, Belastung und zum Arbeits-Erholungszyklus (pp. 46-67). Weinheim: PVU.

Kallus, K. W., \& Krauth, J. (1995). Nichtparametrische Verfahren zum Nachweis emotionaler Reaktionen [Nonparametric Methods for the Detection of Emotional Reactions]. In G. Debus, G. Erdmann, \& K. W. Kallus (Eds.), Biopsychologie von Stress und 
emotionalen Reaktionen (pp. 23-43). Göttingen: Hogrefe-Verlag.

Kallus, K. W., Eberspächer, H., \& Hermann, H. D. (1992). Systematische, naive und gestörte Regeneration im Sport [Systematic, Naive and Disturbed Regeneration in Sports]. In L. Montada (Ed.), Bericht über den 38. Kongreß der Deutschen Gesellschaft für Psychologie in Trier (pp. 436-437). Göttingen: Hogrefe.

Kallus, K. W., Schmitt, J. A., \& Benton, D. (2005). Attention, Psychomotor Functions and Age. European Journal of Nutrition, 44, 465-484. https://doi.org/10.1007/s00394-005-0584-5

Kanfer, R., \& Ackerman, P. L. (2004). Aging, Adult Development, and Work Motivation. The Academy of Management Review, 29, 440-458.

Kellmann, M., \& Kallus, K. W. (2000). Der Erholungs-Belastungsfragebogen für Sportler (EBF-Sport)_Manual [Recovery-Stress Questionnaire for Athletes]. Frankfurt: Swets Test Service.

Kiss, P., De Meester, M., \& Braeckman, L. (2008). Differences between Younger and Older Workers in the Need for Recovery after Work. International Archives of Occupational and Environmental Health, 81, 311-320.

https://doi.org/10.1007/s00420-007-0215-y

Kleitman, N. (1963). Sleep and Wakefulness. Updated and Expanded Edition in Midway Reprints. Chicago and London: The University of Chicago Press (first edition: 1939).

Müller, P. (2011). Einsatz älterer Menschen zur Reduktion des Fachkräftemangels: Eine Analyse in mittelständischen Unternehmen [Use of Elderly People to Reduce Skilled Labor: An Analysis in Medium-Sized Enterprises]. Wiesbaden: Gabler.

Rothgangel, S. (2010). Kurzlehrbuch Medizinische Psychologie und Soziologie [Medical Psychology and Sociology]. Stuttgart: Thieme. https://doi.org/10.1055/b-002-50998

Rothwell, W. J., Sterns, H. L., Spokus, D., \& Reaser, J. M. (2008). Working Longer: New Strategies for Managing, Training, and Retaining Older Employees. New York: AMACOM.

Semmer, N., \& Richter, P. (2004). Leistungsfähigkeit, Leistungsbereitschaft und Belastbarkeit älterer Menschen: Befunde und Konsequenzen [Efficacy, Willingness to Perform and Resilience of Older People: Findings and Consequences]. In M. von Cranach, H. D. Schneider, E. Ulich, \& R. Winkler (Eds.), Ältere Menschen in Unternehmen. Chancen, Risiken, Modelle (pp. 95-116). Bern: Haupt.

Siegrist, J., Wahrendorf, M., von dem Knesebeck, O., Jürges, H., \& Börsch-Supan, A. (2006). Quality of Work, Well-Being, and Intended Early Retirement of Older Employees-Baseline Results from the SHARE Study. European Journal of Public Health, 17, 62-68. https://doi.org/10.1093/eurpub/ckl084

Sluiter, I. K., Van der Beek, A. I., \& Frings-Dresen, M. H. (1999). The Influence of Work Characteristics on the Need for Recovery and Experienced Health: A Study on Coach Drivers. Ergonomics, 42, 573-583. https://doi.org/10.1080/001401399185487

Sonnentag, S., \& Bayer, U.-V. (2005). Switching off Mentally: Predictors and Consequences of Psychological Detachment from Work during Off-Job Time. Journal of Occupational Health Psychology, 10, 393-414. https://doi.org/10.1037/1076-8998.10.4.393

Sonnentag, S., Binnewies, C., \& Mojza, E. J. (2008). “Did You Have a Nice Evening?” A Day-Level Study on Recovery Experience, Sleep, and Affect. Journal of Applied Psychology, 93, 674-684. https://doi.org/10.1037/0021-9010.93.3.674

Statistik Austria (2014). Altersstruktur der Erwerbspersonen 2012, 2030 und 2050 nach Bundesländern (laut Hauptszenario) [Age Structure of the Employees in 2012, 2030 and 2050 by Federal Government (According to the Main Scenario)]. Wien.

Sust, C. A. (1996). Auswirkungen von Geräuschen mittlerer Intensität auf Büro- und 
Verwaltungsaufgaben [Effects of Medium-Intensity Noise on Office and Administrative Tasks]. In Bundesanstalt für Arbeitsschutz und Arbeitsmedizin (Eds.), Lärmbeurteilung-Steuerungs- und Überwachungstätigkeiten. Arbeitswissenschaft-liche Erkenntnisse (pp. 1-11). Dortmund: Bundesanstalt für Arbeitsschutz und Arbeitsmedizin.

Trougakos, J. P., \& Hideg, I. (2009). The Role of Within-Day Work Breaks. In S. Sonnentag, P. L. Perrewé, \& D. C. Ganster (Eds.), Current Perspectives on Job-Stress Recovery Research in Occupational Stress and Well Being (pp. 37-84). Bingley, UK: Emerald Group Publishing Limited. https://doi.org/10.1108/S1479-3555(2009)0000007005

Wagner, V., Kallus, K. W., Jiménez, P., \& Kellmann, M. (2016). The Recovery-Stress Questionnaires: Overall Summary and Hints for Application. In K. W. Kallus, \& M. Kellmann (Eds.), The Recovery-Stress Questionnaires: User Manual (pp. 222-240). Frankfurt am Main: Pearson Assessment \& Information GmbH.

Watson, D. (1988). Intraindividual and Interindividual Analyses of positive and Negative Affect: Their Relation to Health Complaints, Perceived Stress, and Daily Activities. Journal of Personality and Social Psychology, 54, 1020-1030. https://doi.org/10.1037/0022-3514.54.6.1020

Wild-Wall, N., Gajewski, P., \& Falkenstein, M. (2009). Kognitive Leistungsfähigkeit älterer Arbeitnehmer [Cognitive Performance of Older Workers]. Zeitschrift für Gerontologie und Geriatrie, 42, 299-304. https://doi.org/10.1007/s00391-009-0045-5

World Health Organization (WHO) (1993). Aging and Working Capacity. Geneva: World Health Organization Technical Report Series 835.

Zacher, H., Jimmieson, N. L., \& Bordia, P. (2014). Time Pressure and Coworker Support Mediate the Curvilinear Relationship Between Age and Occupational Well-Being. Journal of Occupational Health Psychology, 19, 462-475. https://doi.org/10.1037/a0036995

Zulley, J., \& Knab, B. (2003). Unsere Innere Uhr [Our Inner Clock]. Freiburg: Herder.

\section{Submit or recommend next manuscript to SCIRP and we will provide best} service for you:

Accepting pre-submission inquiries through Email, Facebook, LinkedIn, Twitter, etc. A wide selection of journals (inclusive of 9 subjects, more than 200 journals)

Providing 24-hour high-quality service

User-friendly online submission system

Fair and swift peer-review system

Efficient typesetting and proofreading procedure

Display of the result of downloads and visits, as well as the number of cited articles

Maximum dissemination of your research work

Submit your manuscript at: http://papersubmission.scirp.org/

Or contact psych@scirp.org 